International Journal of Pure and Applied Mathematics

Volume 107 No. 1 2016, 139-143

ISSN: 1311-8080 (printed version); ISSN: 1314-3395 (on-line version)

url: http://www.ijpam.eu

doi: 10.12732/ijpam.v107i1.11

\title{
MATHEMATICAL MODEL OF OPEN QUEUING SYSTEM WITH FULL SET OF MEMORIES
}

\author{
Alexander Kirpichnikov ${ }^{1}$, Anton Titovtsev ${ }^{2} \S$ \\ ${ }^{1,2}$ Kazan National Research Technological University \\ 68, K. Marksa, Kazan, 420015, RUSSIA
}

\begin{abstract}
The paper presents a mathematical model of an open multi-channel queuing system having $m$ service facilities of identical efficiency with exponentially distributed service time. The input stream of Poisson character includes demands of different types arriving from an arbitrary quantity of sources $h$ and having various size-limited queues, at the same time intervals between the nearest-neighbor limits are equal to the unit everywhere. General mathematical formulae for probabilistic characteristics, as well as for the first and second moments of numerical characteristics specifying the quality of service in a steady-state mode of work have been obtained.
\end{abstract}

AMS Subject Classification: 60K25

Key Words: queue, quality of service (QoS), queuing system (QS), M/M/m/K, service facility

\section{Introduction}

In the work of authors [3] the developing idea [1], [2], [4] was presented by the universal mathematical model of an open multi-channel system of queuing having $m$ service facilities of identical efficiency with exponentially distributed service time. A demand input stream in this case is a superposition of components' random number $h$, each of which represents a Poisson stream of claims served in

Received: March 13, 2016

Published: March 25, 2016

${ }^{\S}$ Correspondence author (c) 2016 Academic Publications, Ltd.

url: www.acadpubl.eu 
the order of arrival. For each type of demands entering the system from the $\mathrm{j}$-th source there is a specific size-limited queue $\varepsilon_{j}$ where $\varepsilon_{0}<\varepsilon_{1}<\varepsilon_{2}<\cdots<\varepsilon_{h}$. Quite a detailed conclusion of the main numerical characteristics of discrete and continuous quantities specifying queuing systems of this type was given in the work [3].

From the point of view of possible applications, one more variant of queuing system of this type is worth considering; it differs from the universal model described above by the fact that intervals between the nearest-neighbor limits make a unit everywhere. Thereby, the system contains a full set of all possible sizes of the memory passed with a single step. Thus, this QS variant can be called a queuing system with full set of memories.

This model supplements the universal model in a good way; it has simpler mathematical formulae for various numerical characteristics specifying the quality of QS service, and is convenient for use in cases where size limited queues for claims arriving from different sources differ slightly, within several units.

In this case it is possible to consider formally that the number of sources is more than it is in fact; claims of each successive source have a size-limited queue that is one unite larger than claims from the previous source. At the same time if there is the $\mathrm{j}$-th source of claims for which there is a limitation $\varepsilon_{j}$, then the corresponding demand stream intensity is $\lambda_{j}$, otherwise it is necessary to define the intensity of $\lambda_{j}=0$ stream in a mathematical model.

\section{Probabilistic Characteristics of a Queuing System in a Steady-State Mode}

Let us accept the following system of designations:

$$
\varepsilon_{0}=E_{0}=0 ; \varepsilon_{j}=\sum_{i=0}^{j} E_{i}=j, j=\overline{1, h} ; \text { - a size-limited queue for claims of }
$$

the $\mathrm{j}$-th component;

$$
\begin{aligned}
& \Lambda_{i}=\sum_{j=i}^{h} \lambda_{j} \text {; where } \lambda_{j} \text { - claim stream intensity of the } \mathrm{j} \text {-th component } \\
& R_{i}=\sum_{j=i}^{h} \rho_{j}=\frac{\Lambda_{i}}{\mu} \text {, where } \rho_{j}=\frac{\lambda_{j}}{\mu}-\text { the given claims stream intensity of the }
\end{aligned}
$$
$\mathrm{j}$-th component; $\mu$-claims service intensity carried out by one service facility.

In this case, just as in the work [3], having made up and solved the set of Kolmogorov-Chapman equations of the continuous-time Markov chain with normalization condition $\sum_{i=0}^{m+\varepsilon_{h}} P_{i}=1$ in view, it is possible to obtain the following 
expressions for probabilistic QS states in a steady-state mode of its functioning:

$$
\begin{gathered}
P_{0}=\left[e_{m}\left(R_{0}\right)+\left.\frac{R_{0}^{m}}{m !} \sum_{g=1}^{h} \prod_{j=1}^{g} \frac{R_{j}}{m}\right|^{-1} ;\right. \\
P_{i}=\left\{\begin{array}{l}
\frac{R_{0}^{i}}{i !} P_{0}, \quad 0 \leq i \leq m \\
i-m \\
\prod_{g=1}^{i-m} \frac{R_{g}}{m} \frac{R_{0}^{m}}{m !} P_{0}, \quad m+1 \leq i \leq m+h
\end{array} .\right.
\end{gathered}
$$

In these expressions, as well as in [3], $e_{m}\left(R_{0}\right)=\sum_{i=0}^{m} \frac{R_{0}^{i}}{i !}$ is an incomplete exponential. Hence, traditional probabilistic characteristics of QS service:

- probability of an immediate service of a newly arrived claim in the system $P_{I S}=\sum_{i=0}^{m-1} P_{i}=e_{m-1}\left(R_{0}\right) P_{0}$

- probability of a newly arrived claim service expectation in the queue $P_{W}=$ $\frac{1}{R_{0}} \sum_{i=1}^{h} R_{i} P_{m+i-1}$

- probability of a newly arrived claim service refusal

$$
\begin{gathered}
P_{L}=\frac{1}{R_{0}} \sum_{i=1}^{h}\left(R_{0}-R_{i}\right) P_{m+i-1}+P_{m+h}= \\
=\sum_{i=1}^{h} P_{m+i-1}-P_{W}+P_{m+h}=1-P_{I S}-P_{W} .
\end{gathered}
$$

\section{Numerical Characteristics of a Queuing System}

By means of the probabilistic characteristics found above and in compliance with the technique, it is possible to express easily all main numerical characteristics of a steady-state mode of QS work of this type. We have

- throughout capacity of a queuing system:

$$
A=\Lambda_{0}\left(1-P_{L}\right)=\Lambda_{0}\left(P_{I S}+P_{W}\right) ;
$$

- moments of demands number under service at the same time:

$$
n^{(1)}=R_{0} P_{0} e_{m-2}\left(R_{0}\right)+m\left(P_{W}+P_{L}\right) ;
$$




$$
n^{(2)}=R_{0} P_{0} e_{m-2}\left(R_{0}\right)+R_{0}^{2} P_{0} e_{m-3}\left(R_{0}\right)+m^{2}\left(P_{W}+P_{L}\right) ;
$$

- moments of claims number in queue for service:

$$
l^{(K)}=\sum_{i=m+1}^{m+h}(i-m)^{K} \prod_{g=1}^{i-m} \frac{R_{g}}{m} \frac{R_{0}^{m}}{m !} P_{0}, K=1,2
$$

- moments of demands number in the system on the whole:

$$
k^{(K)}=\sum_{i=1}^{m} i^{K} \frac{R_{0}^{i}}{i !} P_{0}+\sum_{i=m+1}^{m+h} i^{K} \prod_{g=1}^{i-m} \frac{R_{g}}{m} \frac{R_{0}^{m}}{m !} P_{0}, K=1,2 .
$$

- distribution function of claim waiting time in a queue:

$$
F_{W}(t)=1-\frac{m}{q R_{0}} e^{-m \mu t} \sum_{i=1}^{h} P_{m+i} e_{i-1}(m \mu t) ;
$$

- density of claim waiting time distribution in a queue

$$
f_{W}(t)=\frac{d F_{W}(t)}{d t}=\frac{m^{2} \mu^{2}}{q \Lambda_{0}} e^{-m \mu t} \sum_{i=1}^{h} P_{m+i} \frac{(m \mu t)^{i-1}}{(i-1) !}
$$

- moments of expected waiting time of claim service in a queue:

$$
t_{W}^{(1)}=l^{(1)} / A ; t_{W}^{(2)}=\frac{1}{A m \mu} \sum_{i=m+1}^{m+h}(i-m)(i-m+1) P_{i}
$$

- moments of time when a claim is staying in the system on the whole:

$$
\begin{gathered}
t_{T}^{(1)}=k^{(1)} / A \\
t_{T}^{(2)}=\frac{1}{A m \mu}\left[\sum_{i=m+1}^{m+h}(i-m)(i-m+1) P_{i}+2 m \sum_{i=1}^{m+h} i P_{i}\right] .
\end{gathered}
$$

\section{References}

[1] J.W. Cohen, Certain delay problems for a full availability trunk group loaded by two sources, Communication News, 16, No. 3 (1956), 105-113.

[2] A.P. Kirpichnikov, A.S. Titovtsev, Open systems of multicomponent flows differentiated service, Ciência e Técnica Vitivinícola, 29, No. 7 (2014), 108-122. 
[3] A. Kirpichnikov, A. Titovtsev, Mathematical model of a queuing system with arbitrary quantity of sources and size-limited queue, International Journal of Pure and Applied Mathematics, 106, No. 2 (2016), 649-661.

[4] H. Takagi, Explicit delay distribution in first-come first-served $M / M / m / K$ and $\mathrm{M} / \mathrm{M} / \mathrm{m} / \mathrm{K} / \mathrm{n}$ queues and mixed loss-delay system, International Journal of Pure and Applied Mathematics, 40, No. 2 (2007), 185-200. 
